\title{
Stage IIIA Choroidal and Ciliary Body Melanoma AJCC v8
}

National Cancer Institute

\section{Source}

National Cancer Institute. Stage IIIA Choroidal and Ciliary Body Melanoma A/CC V8. NCI

Thesaurus. Code C140665.

Stage IIIA includes: (T2c-d, N0, M0); (T3b-c, N0, M0); (T4a, NO, MO). T2c: Tumor size category 2 without ciliary body involvement but with extraocular extension less than or equal to $5 \mathrm{~mm}$ in largest diameter. T2d: Tumor size category 2 with ciliary body involvement and extraocular extension less than or equal to $5 \mathrm{~mm}$ in largest diameter. T3b: T umor size category 3 with ciliary body involvement. T3c: T umor size category 3 without ciliary body involvement but with extraocular extension less than or equal to 5 $\mathrm{mm}$ in largest diameter. T4a: T umor size category 4 without ciliary body involvement and extraocular extension. N0: No regional lymph node metastasis. M0: No distant metastasis. (AJCC 8th ed.) 